\title{
Pain and quality of life in knee osteoarthritis, chronic low back pain and fibromyalgia: a comparative cross-sectional study
}

\author{
M. de Oliveira Paes Leme, S.L.K. Yuan, M. Oliveira Magalhães, \\ S.R. Ferreira de Meneses, A.P. Marques \\ Department of Physical therapy, Speech Therapy and Occupational Therapy of the School of Medicine, \\ University of Sao Paulo, Brazil
}

\section{SUMMARY}

The objective of our study was to compare pain and health-related quality of life among patients with knee osteoarthritis (OA), chronic nonspecific low back pain (CNLBP) and fibromyalgia (FM).

This cross-sectional study included 87 subjects, who were divided into three groups according to their diagnosis: knee OA ( $n=29)$, CNLBP ( $n=29)$ and FM (n=29), between March 2013 and March 2014. Pain intensity was measured using the Numeric Pain Rating Scale (NPRS), quality of pain using the McGill Pain Questionnaire (MPQ) and health-related quality of life using the 36-item Short-Form Health Survey (SF-36). Painful body areas were marked on a pain map.

No statistically significant differences between groups were found for NPRS. Regarding MPQ, the knee OA group presented a lower pain-rating index in contrast to the CNLBP and FM groups, and no significant differences were found between the CNLBP and FM groups. A greater number of characteristic pain descriptors and painful locations were found in the FM group than in the CNLBP or knee OA groups. Regarding SF-36, the FM group presented statistically significant lower values for bodily pain in contrast to the knee OA group. Even though the global pain intensity was similar between groups, the findings suggest that the FM group presented the worst pain experience and a lower health-related quality of life than the knee OA group in terms of bodily pain. They also suggest that the pain experience was worse for the CNLBP group than for the knee OA group but health-related quality of life was similar.

Key words: Osteoarthritis; low back pain; fibromyalgia; pain measurement; quality of life.

\section{INTRODUCTION}

hronic pain is defined as pain that persists past the normal time of healing. Three months has been suggested as the most convenient cut-off between acute and chronic pain $(1,2)$. According to the biopsychosocial model, chronic pain is the subjective experience of nociception, and results from the complex interaction of biological changes, psychological status and sociocultural context (3). The prevalence of chronic pain in the general population is high. A systematic review, which included 19 studies in 34 countries, estimated a worldwide prevalence of $30.3 \%$ (4). Chronic pain is a major health problem, which seriously affects the patients' daily activities, social and working lives, and poses an economic burden on patients, healthcare services and society (5).

Osteoarthritis (OA), chronic nonspecific low back pain (CNLBP) and fibromyalgia (FM) are highly prevalent conditions and among the leading reasons why patients seek medical care (6). Review articles found pooled OA prevalence which ranged from $10.9 \%$ to $43.3 \%$ depending on the joint site (7), mean chronic low back pain (LBP) prevalence of $20.1 \%$ (8), and FM prevalence which ranged from $0.2 \%$ to $6.6 \%$ in the general population (9).

OA is the most common form of arthritis, characterized by abnormal remodelling of joint tissues driven by a host of inflammatory mediators within the affected joint. 
The pathologic changes seen in OA joints include damage of the articular cartilage, thickening of the subchondral bone, osteophyte formation, variable degrees of synovial inflammation, degeneration of ligaments and, in the knee, the menisci, and hypertrophy of the joint capsule (10). CNLBP is defined as chronic pain located in the posterior aspect of the body from the lower margin of the $12^{\text {th }}$ ribs to the lower gluteal folds (8). There is strong evidence that CNLBP is associated with a complex interaction of factors, which include physical, cognitive, psychological, lifestyle, neurophysiological and social ones (11). FM is currently considered a central sensitivity syndrome. The hallmark symptom of FM is chronic widespread pain, which cannot be adequately explained on the basis of the degree of peripheral tissue inflammation or damage. It is usually associated with other symptoms such as fatigue, sleep disorders, headache, irritable bowel syndrome and mood disorders (12).

Pain is a universal, subjective experience, and may be defined as an unpleasant sensory and emotional experience associated with actual or potential tissue damage, or described in terms of such damage. It can serve as a warning to protect us from further harm, but it can also contribute to persistent suffering, when it surpasses its underlying cause to become a disease in its own domains and dimensions. Pain is a complex phenomenon, in which the unique way each individual perceives pain and its severity depends on a constellation of biological, psychological and social factors. It is important to realize that emotional and psychosocial reactions to pain are clinically significant (13).

Therefore, even though it is common to use unidimensional measures of global intensity such as the visual analogue and the numeric rating scales, a comprehensive assessment of chronic pain requires a multidimensional approach to capture fully the patient's pain experience in OA, CNLBP and FM, among other conditions. Other features of pain should be assessed besides intensity: quality of pain (e.g. sharp, burning, sensitive), temporal aspects (e.g. dura- tion, frequency) and spatial characteristics (e.g. location of pain) (14). Since chronic pain has a negative impact on quality of life, an evaluation of different aspects of quality of life should also be included (5). Knowledge of the pain characteristics and the impact on quality of life of each chronic pain condition is relevant to adequate pain assessment, optimal pain management and accurate evaluation of treatment outcome in clinical and research settings. Hence, this study aimed to assess and compare pain and quality of life among patients with knee OA, CNLBP and FM.

\section{MATERIALS AND METHODS}

\section{Study design and sample}

A cross-sectional study was conducted at the Rehabilitation Center in Taboao da Serra, SP, Brazil, between March 2013 and March 2014. Adult patients (18 years and older) with chronic pain were recruited systematically by clinical referral and allocated into three groups according to their diagnosis: knee OA ( $\mathrm{n}=29)$, CNLBP $(n=29)$ and FM $(n=29)$. The study was approved by the research ethics committee of the School of Medicine at the University of Sao Paulo (protocol number 393/12). Informed consent was obtained from all participants included in the study.

For the knee OA group, participants were included according to the American College of Rheumatology (ACR) clinical classification criteria (the combination of pain in the knee and at least three of the following: over 50 years of age, less than 30 minutes of morning stiffness, crepitus on active motion, bony tenderness, bony enlargement and no palpable warmth of synovium) (15). For the CNLBP group, inclusion criteria were the presence of pain of unknown origin, localized below the costal margin and above the inferior gluteal folds, with or without leg pain, for more than 3 months (16). For the FM group, participants were included according to the 1990 ACR classification criteria for FM (the combination of chronic widespread pain with the presence of at least 11 of 18 tender points) (17). 
Exclusion criteria were: severe cognitive impairment, psychiatric disorders, pregnancy, previous surgeries, sensory or motor neurological deficit, severe musculoskeletal disorders (infection, fracture or inflammation), cancer, neuropathic pain and other rheumatic diseases.

\section{Assessment procedures}

Each participant underwent a comprehensive assessment of pain and quality of life using the following tools: Numeric Pain Rating Scale (NPRS), McGill Pain Questionnaire (MPQ), Pain Map and 36-item Short-Form Health Survey (SF-36).

The NPRS is a valid and reliable unidimensional measure of pain intensity in adults, including those with chronic pain due to rheumatic diseases. It is an 11-point numeric scale on which the respondent marks the number that best reflects the intensity of their pain in the last $24 \mathrm{~h}$. Scores range from 0 to 10 , and higher scores indicate greater pain intensity (18).

The MPQ is a valid multidimensional pain questionnaire designed to assess the quality of pain in adults with chronic pain, including pain due to rheumatic diseases $(18,19)$. The Brazilian version of the MPQ contains 77 pain descriptors categorized into 20 subclasses that fall into four major groups: sensory, affective, evaluative and miscellaneous $(20,21)$. The score associated with each descriptor is based on its rank order within the word set. Rank values are summed within each major group (sensory 0-41, affective $0-14$, evaluative $0-5$, miscellaneous $0-17$ ) as well as overall. Overall score corresponds to the Pain Rating Index, which ranges from 0 to 77. A higher score on the MPQ indicates worse pain. The Pain Rating Index is interpreted both in terms of quantity of pain, as evidenced by the number of words used and the rank values of the words, as well as the quality of pain, as evidenced by the particular words that are chosen.

The Pain Map was used in order to obtain information about the location of pain on the body. It corresponds to outlines of a human figure (front and back) on which participants mark the areas where they are experiencing pain at the moment. The Pain
Map is divided into 45 body areas (22). The SF-36 is a 36-item survey intended to measure health-related quality of life. It is suitable for use in general, as well as in clinical populations, and it has been frequently used in a variety of rheumatic conditions (23-25). The SF-36 measures eight domains: physical functioning, role physical, bodily pain, general health, vitality, social functioning, role emotional and mental health.

The crude scores for each domain are calculated and transformed into scales that range from 0 to 100 , with higher scores indicating better quality of life.

\section{Statistical analysis}

Groups were compared with one-way analysis of covariance (ANCOVA) and the post-hoc LSD test. Means were adjusted for age and gender. Statistical analysis was performed using SPSS Statistics 19 (IBM, Armonk, United States). A significance level of 0.05 was adopted.

\section{RESULTS}

The sample consisted of 87 participants, mostly women $(85.1 \%)$ and married (58.6\%), aged between 25 and 80 years old, with a mean age of $62.6 \pm 8.7$ for the knee OA group, $48.4 \pm 11.8$ for the CNLBP group and 57.8 \pm 11.2 for the FM group. A higher proportion of participants worked outside the home in the CNLBP (69\%) and FM (55.2\%) in comparison to the knee OA group $(31 \%)$.

Table I shows the quantitative data of pain and quality of life of the three groups. No statistically significant differences between groups were found for the NPRS. The knee OA group presented lower values than the CNLBP group, with statistical significance, for the sensory dimension and the pain-rating index of MPQ. No differences between the knee OA and CNLBP groups were found for SF-36. The knee OA group presented lower values than FM group, with statistical significance, for the sensory, affective and miscellaneous dimensions and the pain-rating index of MPQ. Regarding SF-36, the knee OA group presented higher 
Table I - Pain and health-related quality of life in knee osteoarthritis, chronic nonspecific low back pain and fibromyalgia groups. Means adjusted for age and gender.

\begin{tabular}{|l|c|c|c|c|}
\hline & $\begin{array}{c}\text { Knee OA Group } \\
\text { Mean (95\% CI) }\end{array}$ & $\begin{array}{c}\text { CNLBP Group } \\
\text { Mean (95\% Cl) }\end{array}$ & $\begin{array}{c}\text { FM Group } \\
\text { Mean (95\% CI) }\end{array}$ & $\begin{array}{c}\text { ANCOVA } \\
\text { p-value }\end{array}$ \\
\hline Pain & $6.4(5.1,7.5)$ & $4.7(3.4,6.0)$ & $6.3(5.3,7.6)$ & 0.123 \\
\hline NPRS & $20.0(17.6,22.5)^{\star} \#$ & $25.0(22.4,27.5)^{\star}$ & $23.6(21.2,25.9) \#$ & 0.025 \\
\hline MPQ - Sensory & $4.1(2.9,5.4)^{\star}$ & $6.0(4.7,7.4)$ & $6.9(5.7,8.1)^{\star}$ & 0.010 \\
\hline MPQ - Affective & $2.6(1.9,3.2)$ & $2.6(1.9,3.3)$ & $3.3(2.7,3.9)$ & 0.185 \\
\hline MPQ - Evaluative & $6.6(5.4,7.8) \#$ & $7.4(6.1,8.7)^{\star}$ & $9.3(8.2,10.5)^{\star} \#$ & 0.005 \\
\hline MPQ - Miscellaneous & $33.3(28.7,37.8)^{\star} \#$ & $41.1(36.3,45.8)^{\star}$ & $42.7(38.4,47.0) \#$ & 0.010 \\
\hline MPQ - Pain Rating Index & & & \\
\hline Quality of life (SF-36) & $56.1(45.3,66.8)$ & $63.7(52.5,74.9)$ & $46.5(36.2,56.8)$ & 0.091 \\
\hline Physical functioning & $44.6(27.5,61.7)$ & $46.7(28.9,64.4)$ & $38.9(22.6,55.3)$ & 0.800 \\
\hline Role physical & $55.3(45.7,64.9)^{\star}$ & $48.0(38.0,57.9)$ & $35.2(27.0,45.4)^{\star}$ & 0.017 \\
\hline Bodily pain & $67.0(58.0,76.1)$ & $64.6(55.2,74.0)$ & $52.9(44.2,61.6)$ & 0.055 \\
\hline General health & $67.1(56.5,77.6)$ & $64.8(53.9,75.8)$ & $54.7(44.6,64.8)$ & 0.192 \\
\hline Vitality & $80.0(66.4,93.6)$ & $76.2(62.0,90.3)$ & $69.0(56.0,82.1)$ & 0.491 \\
\hline Social functioning & $73.1(57.3,88.8)$ & $90.8(74.5,107.2)$ & $66.0(50.9,81.1)$ & 0.101 \\
\hline Role emotional & $73.5(63.2,83.8)$ & $74.0(63.3,84.6)$ & $58.9(49.1,68.7)$ & 0.058 \\
\hline Mental health &
\end{tabular}

OA, osteoarthritis; CNLBP, chronic nonspecific low back pain; FM, fibromyalgia; ANCOVA, analysis of covariance; Cl, confidence interval; NPRS, numeric pain rating scale; MPQ, McGill pain questionnaire; SF36, 36-item short-form health survey. * \# difference between pairs was statistically significant.

Table II - Frequency (\%) of McGill pain questionnaire descriptors in knee osteoarthritis, chronic nonspecific low back pain and fibromyalgia groups.

\begin{tabular}{|c|c|c|c|c|c|c|}
\hline & \multicolumn{2}{|c|}{ Knee OA Group } & \multicolumn{2}{|c|}{ CNLBP Group } & \multicolumn{2}{|c|}{ FM Group } \\
\hline & Descriptors & $\%$ & Descriptors & $\%$ & Descriptors & $\%$ \\
\hline \multirow[t]{7}{*}{ Sensory } & Shooting & 62 & Aching & 70 & Beating & 62 \\
\hline & Tugging & 62 & Beating & 62 & Flashing & 59 \\
\hline & Sharp & 48 & Flashing & 55 & Cramping & 52 \\
\hline & Heavy & 41 & Wrenching & 52 & Taut & 49 \\
\hline & & & Burning & 49 & Sharp & 45 \\
\hline & & & & & Tugging & 45 \\
\hline & & & & & Aching & 45 \\
\hline \multirow[t]{3}{*}{ Affective } & Tiring & 66 & Tiring & 62 & Sickening & 73 \\
\hline & & & Sickening & 52 & Tiring & 62 \\
\hline & & & & & Blinding & 52 \\
\hline \multirow[t]{4}{*}{ Miscellaneous } & Spreading & 55 & Dreadful & 55 & Spreading & 49 \\
\hline & & & Spreading & 52 & Dreadful & 48 \\
\hline & & & & & Tight & 45 \\
\hline & & & Miserable & 41 & & \\
\hline
\end{tabular}

MPQ, McGill pain questionnaire; OA, osteoarthritis; CNLBP, chronic nonspecific low back pain; FM, fibromyalgia. 


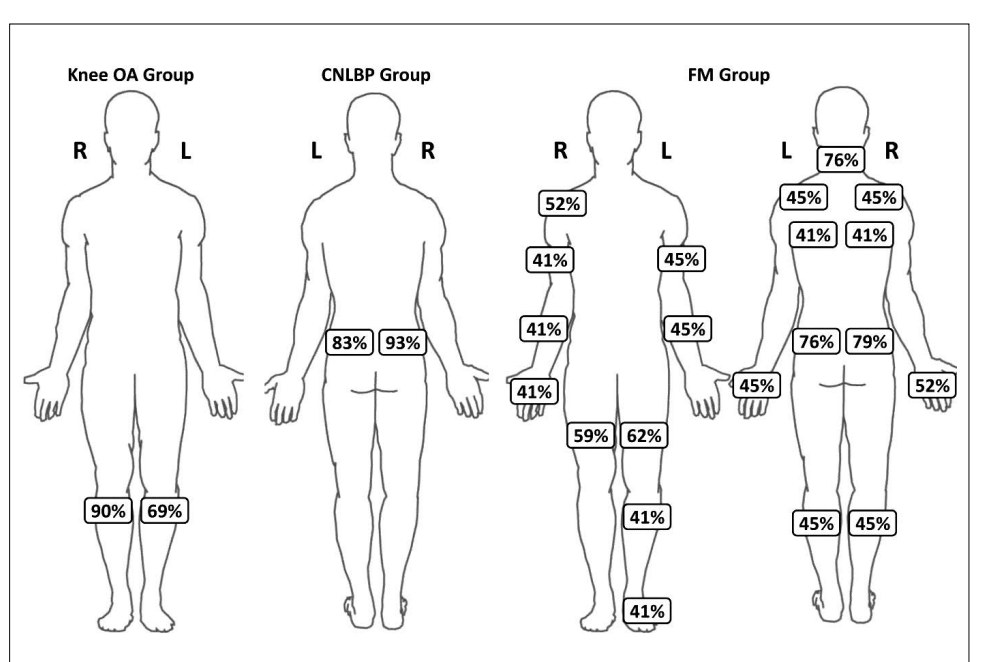

Figure 1 - Spatial distribution of pain in knee osteoarthritis, chronic nonspecific low back pain and fibromyalgia groups.

values than the FM group for bodily pain. The CNLBP group presented lower values than the FM group for the miscellaneous dimension of MPQ. No significant differences were found between the CNLBP and FM groups for SF-36.

Table II shows the MPQ descriptors that were selected at a relative frequency greater than $40 \%$. The FM Group had the greatest number of descriptors $(n=13)$, and the knee OA Group the lowest $(n=6)$. Two descriptors were observed in all groups: tiring and spreading. The descriptor selected at the highest frequency was sickening in the FM Group.

The spatial distribution of pain is presented in Figure 1, with data of the most frequently selected areas $(>40 \%)$ across the three groups.

\section{DISCUSSION}

The purpose of the study was to perform a comprehensive assessment of the patients' pain experience and compare pain characteristics and quality of life among groups of participants with knee OA, CNLBP and FM. Even though no differences were found between groups regarding the quantitative measure of the global intensity of pain, in the analysis of the quality of pain, the findings suggest that the FM group presented the worst pain experience, and that the pain experience for the CNLBP group was worse than for the knee OA group. Regarding the spatial distribution, pain was focused in knee OA and CNLBP in two body areas (right/left anterior leg, and right/left lumbar, respectively) while pain was widespread in FM most frequently across 21 out of a total of 45 areas. For the FM group, health-related quality of life was slightly poorer in contrast to the knee OA group, in terms of bodily pain. No significant differences were found regarding health-related quality of life between the knee OA and CNLBP groups.

A post-hoc analysis of placebo-controlled trials included three clinical trials with knee OA and two with chronic LBP. The authors investigated the effects of duloxetine on pain intensity as the primary outcome, measured by the NPRS. At baseline, they found that patients with knee OA and chronic LBP were similar regarding pain intensity (26). A comparative study of prospective and recalled pain assessments in LBP and lower limb OA used the visual analogue scale and found that the mean baseline pain intensity was similar between groups (27). A cross-sectional study compared pain intensity using a visual analogue scale in patients with FM and OA (spine, hip or knee). Differently from the present study, the authors observed higher pain intensity for FM than OA (28). No studies that compare pain intensity in FM and LBP were found.

A cross-sectional study compared the quality of pain among patients with FM, OA and LBP, using the MPQ sensory and affective classes. Differently from the present study, groups were similar regarding the sensory class.

Regarding the affective class, FM was significantly worse than both OA and LBP. No differences were found between OA and LBP. Concerning the number of the most characteristic descriptors for FM, 18 were mentioned by at least a third of the subjects, of which seven are common to the present study: flashing, sharp, tugging, aching, sickening, tiring and blinding. For $\mathrm{OA}$, there were 11 characteristic descriptors, of which only two were in common 
with this study: sharp and tiring. For LBP, there were also 11 descriptors, of which four were common: burning, wrenching, sickening and tiring (29).

Research has consistently documented the relationship between the presence of chronic pain and poor health-related quality of life (25). A previous cross-sectional study compared health-related quality of life between patients with FM and knee OA. The authors observed differences in more domains than the present study: the FM group presented lower scores for bodily pain, general health, vitality and social functioning than the knee OA group. Differently from this study, the FM group presented a higher score for physical functioning than the OA group (30). Another study used SF-36 to compare patients with FM and knee OA and found that both groups were similar, except for physical functioning, which was higher for FM, and general health, which was higher for knee OA (31). A prospective study compared a group of patients with arthritis (rheumatoid arthritis or knee, hip, hand, wrist $\mathrm{OA}$ ) and a group of patients with LBP, using SF-36. At baseline, no differences were found between groups, except for general health, which was lower for the arthritis group (32). No studies that used SF-36 as a measure of healthrelated quality of life to compare patients with OA and LBP or patients with FM and LBP were identified.

Even though the global pain intensity was similar between groups, findings suggest that the FM group presented the worst pain experience, based on the quality, spatial distribution and impact of pain on health-related quality of life. The quality of pain was worse for the CNLBP group than the OA group, but health-related quality of life was similar. In order to capture better the patient's pain experience across different diagnoses, a more comprehensive pain assessment is required, which could include an analysis of quality of pain, spatial distribution and impact on different domains of health-related quality of life besides the global measure of pain intensity.

\section{REFERENCES}

1. Merskey H, Bogduk N, International Association for the Study of Pain. Task Force on Taxonomy. Classification of chronic pain: descriptions of chronic pain syndromes and definitions of pain terms. 2nd ed. Seattle: IASP Press; 1994.

2. Chou R, Qaseem A, Snow V, et al. Diagnosis and treatment of low back pain: a joint clinical practice guideline from the American College of Physicians and the American Pain Society [Practice Guideline Research Support, Non-U.S. Gov't]. Ann Intern Med. 2007; 147: 478-91.

3. Gatchel RJ, Peng YB, Peters ML, et al. The biopsychosocial approach to chronic pain: scientific advances and future directions [Research Support, N.I.H., Extramural Research Support, U.S. Gov't, Non-P.H.S. Review]. Psychol Bull. 2007; 133: 581-624.

4. Elzahaf RA, Tashani OA, Unsworth BA, et al. The prevalence of chronic pain with an analysis of countries with a Human Development Index less than 0.9: a systematic review without meta-analysis [Review]. Curr Med Res Opin. 2012; 28: 1221-9.

5. Breivik H, Collett B, Ventafridda V, et al. Survey of chronic pain in Europe: prevalence, impact on daily life, and treatment [Research Support, Non-U.S. Gov't]. Eur J Pain. 2006; 10: 287-333.

6. Mody GM, Brooks PM. Improving musculoskeletal health: global issues. Best Pract Res Clin Rheumatol. 2012; 26: 237-49.

7. Pereira D, Peleteiro B, Araujo J, et al. The effect of osteoarthritis definition on prevalence and incidence estimates: a systematic review [Meta-Analysis Review]. Osteoarthr Cartilage. 2011; 19: 1270-85.

8. Hoy D, Bain C, Williams G, et al. A systematic review of the global prevalence of low back pain [Research Support, Non-U.S. Gov't Review]. Arthritis Rheum. 2012; 64: 2028-37.

9. Marques AP, Santo A, Berssaneti AA, et al. Prevalence of fibromyalgia: literature review update. Rev Brasil Reumatol. 2017; 57: 356-63.

10. Loeser RF, Goldring SR, Scanzello CR, et al. Osteoarthritis: a disease of the joint as an organ [Research Support, N.I.H., Extramural Research Support, Non-U.S. Gov't Review]. Arthritis Rheum. 2012; 64: 1697-707.

11. O'Keeffe M, Purtill H, Kennedy N, et al. Individualised cognitive functional therapy compared with a combined exercise and pain education class for patients with non-specific chronic low back pain: study protocol for a multicentre randomised controlled trial [Multicenter Study Randomized Controlled Trial Research Support, Non-U.S. Gov't]. BMJ Open. 2015; 5: e007156. 
12. Cassisi G, Sarzi-Puttini P, Casale R, et al. Pain in fibromyalgia and related conditions [Review]. Reumatismo. 2014; 66: 72-86.

13. Institute of Medicine (U.S.). Committee on Advancing Pain Research Care and Education. Relieving pain in America: a blueprint for transforming prevention, care, education, and research. Washington, DC: National Academies Press; 2011.

14. Jensen MP, Dworkin RH, Gammaitoni AR, et al. Do pain qualities and spatial characteristics make independent contributions to interference with physical and emotional functioning? [Comparative Study Research Support, Non-U.S. Gov't]. J Pain. 2006; 7: 644-53.

15. Altman R, Asch E, Bloch D, et al. Development of criteria for the classification and reporting of osteoarthritis. Classification of osteoarthritis of the knee. Diagnostic and Therapeutic Criteria Committee of the American Rheumatism Association [Guideline Practice Guideline Research Support, U.S. Gov't, P.H.S.]. Arthritis Rheum. 1986; 29: 1039-49.

16. Koes BW, van Tulder MW, Thomas S. Diagnosis and treatment of low back pain [Review]. BMJ. 2006; 332: 1430-4.

17. Wolfe F, Smythe HA, Yunus MB, et al. The American College of Rheumatology 1990 Criteria for the Classification of Fibromyalgia. Report of the Multicenter Criteria Committee [Comparative Study Research Support, Non-U.S. Gov't]. Arthritis Rheum. 1990; 33: 160-72.

18. Hawker GA, Mian S, Kendzerska T, et al. Measures of adult pain: Visual Analog Scale for Pain (VAS Pain), Numeric Rating Scale for Pain (NRS Pain), McGill Pain Questionnaire (MPQ), Short-Form McGill Pain Questionnaire (SF-MPQ), Chronic Pain Grade Scale (CPGS), Short Form-36 Bodily Pain Scale (SF-36 BPS), and Measure of Intermittent and Constant Osteoarthritis Pain (ICOAP) [Review]. Arthritis Care Res (Hoboken). 2011; 63: S240-52.

19. Melzack R. The McGill Pain Questionnaire: major properties and scoring methods [Research Support, U.S. Gov't, Non-P.H.S.]. Pain. 1975; 1: 277-99.

20. Varoli FK, Pedrazzi V. Adapted version of the McGill Pain Questionnaire to Brazilian Portuguese. Braz Dent J. 2006; 17: 328-35.

21. Menezes Costa Lda C, Maher CG, McAuley $\mathrm{JH}$, et al. The Brazilian-Portuguese versions of the McGill Pain Questionnaire were reproducible, valid, and responsive in patients with musculoskeletal pain [Validation Studies]. J Clin Epidemiol. 2011; 64: 903-12.

22. Margolis RB, Tait RC, Krause SJ. A rating system for use with patient pain drawings. Pain. 1986; 24: 57-65.

23. Ware JE, Jr., Sherbourne CD. The MOS 36- item short-form health survey (SF-36). I. Conceptual framework and item selection [Research Support, Non-U.S. Gov't Research Support, U.S. Gov't, P.H.S.]. Med Care. 1992; 30: 473-83.

24. Ciconelli RM, Ferraz MB, Santos W, et al. Brazilian-Portuguese version of the SF-36. A reliable and valid quality of life outcome measure. Rev Bras Reumatol. 1999; 39: 8.

25. Busija L, Pausenberger E, Haines TP, et al. Adult measures of general health and healthrelated quality of life: Medical Outcomes Study Short Form 36-Item (SF-36) and Short Form 12-Item (SF-12) Health Surveys, Nottingham Health Profile (NHP), Sickness Impact Profile (SIP), Medical Outcomes Study Short Form 6D (SF-6D), Health Utilities Index Mark 3 (HUI3), Quality of Well-Being Scale (QWB), and Assessment of Quality of Life (AQoL) [Research Support, Non-U.S. Gov't Review]. Arthritis Care Res (Hoboken). 2011; 63:S383-412.

26. Williamson OD, Schroer M, Ruff DD, et al. Onset of response with duloxetine treatment in patients with osteoarthritis knee pain and chronic low back pain: a post hoc analysis of placebo-controlled trials [Randomized Controlled Trial Research Support, Non-U.S. Gov't]. Clin Ther. 2014; 36: 544-51.

27. Perrot S, Marty M, Legout V, et al. Ecological or recalled assessments in chronic musculoskeletal pain? A comparative study of prospective and recalled pain assessments in low back pain and lower limb painful osteoarthritis [Comparative Study Research Support, NonU.S. Gov't]. Pain Med. 2011; 12: 427-36.

28. Oliveira P, Monteiro P, Coutinho M, et al. [Health-related quality of life and chronic pain experience in rheumatic diseases] [Comparative Study]. Acta Reumatol Port. 2009; 34: 511-9.

29. Marques AP, Rhoden L, de Oliveira Siqueira J, et al. Pain evaluation of patients with fibromyalgia, osteoarthritis, and low back pain [Comparative Study Evaluation Studies]. Rev Hosp Clin Fac Med Sao Paulo. 2001; 56: 5-10.

30. Ozcetin A, Ataoglu S, Kocer E, et al. Effects of depression and anxiety on quality of life of patients with rheumatoid arthritis, knee osteoarthritis and fibromyalgia syndrome. West Indian Med J. 2007; 56: 122-9.

31. Yilmaz F, Sahin F, Ergoz E, et al. Quality of life assessments with SF 36 in different musculoskeletal diseases [Comparative Study]. Clin Rheumatol. 2008; 27: 327-32.

32. Osterhaus JT, Dedhiya SD, Ernst ME, et al. Health outcomes assessment in community pharmacy practices: a feasibility project [Research Support, Non-U.S. Gov't]. Arthritis Rheum. 2002; 47: 124-31. 